\title{
TWO REMARKS ON POLYNOMIALLY BOUNDED REDUCTS OF THE RESTRICTED ANALYTIC FIELD WITH EXPONENTIATION
}

\author{
SERGE RANDRIAMBOLOLONA
}

\begin{abstract}
This article presents two constructions motivated by a conjecture of van den Dries and Miller concerning the restricted analytic field with exponentiation. The first construction provides an example of two o-minimal expansions of a real closed field that possess the same field of germs at infinity of onevariable functions and yet define different global one-variable functions. The second construction gives an example of a family of infinitely many distinct maximal polynomially bounded reducts (all this in the sense of definability) of the restricted analytic field with exponentiation.
\end{abstract}

\section{$\S 1$. Introduction}

Properties of $\mathbb{R}_{\mathrm{an}, \exp }$, the real exponential field with restricted analytic functions, have been widely studied since the mid-1990s (starting with van den Dries and Miller [14] and van den Dries, Macintyre, and Marker [13]).

Of particular interest are the properties of $\mathbb{R}_{\mathrm{an}, \text { Pow }}$, the real field with power functions and restricted analytic functions, which is a reduct, in the sense of definability, of $\mathbb{R}_{\text {an,exp }}$. (Most definitions are not recalled in this section, in order to make the introduction lighter. We assume that the reader is familiar with the terminology of model theory (see, e.g., [8, Chapters 1-5]) and with o-minimality (see, e.g., [12]); less standard notions (such as what we mean by in the sense of definability) are made precise in Sections 2 and 3.) Miller [5] studied the theory of $\mathbb{R}_{\mathrm{an}, \text { Pow }}$ and proved, among other things, that $\mathbb{R}_{\mathrm{an}, \text { Pow }}$ is polynomially bounded (and, in particular, is a proper reduct, in the sense of definability, of $\left.\mathbb{R}_{\mathrm{an}, \exp }\right)$.

Van den Dries and Miller in [15] conjectured that the structure $\mathbb{R}_{\mathrm{an}, \text { Pow }}$ is maximal among the polynomially bounded reducts of $\mathbb{R}_{\text {an,exp }}$ (all this in the sense of definability).

Received March 19, 2013. Revised August 22, 2013. Accepted August 24, 2013.

First published online July 14, 2014.

2010 Mathematics Subject Classification. Primary 03C64; Secondary 32B20. 
An important partial answer was given independently by Soufflet [10, Proposition 5.1] and by Kuhlmann and Kuhlmann [3, Corollary 2]: they proved that if $\mathbb{R}_{\mathcal{F}}$ is a proper reduct, in the sense of definability, of $\mathbb{R}_{\mathrm{an} \text {,exp }}$ that is also an expansion, in the sense of definability, of $\mathbb{R}_{\text {an,Pow }}$, then $\mathbb{R}_{\mathcal{F}}$ and $\mathbb{R}_{\text {an,Pow }}$ define the same subsets of $\mathbb{R}^{2}$. If $\mathbb{R}_{\text {an,Pow }}$ is not maximal among the strict reducts of $\mathbb{R}_{\mathrm{an}, \exp }$ (in the sense of definability), then a set witnessing this nonmaximality needs to be of arity at least 3 .

As was noted by the author in [9], two o-minimal expansions of the real field may define the same subsets of $\mathbb{R}^{2}$, while the first is a strict reduct, in the sense of definability, of the second. However, this phenomenon cannot appear in a saturated setting: [11, Lemma 4.7] ensures that if $R_{\mathcal{L}_{0}}$ is a reduct of $R_{\mathcal{L}_{1}}$, each of the structures $R_{\mathcal{L}_{0}}$ and $R_{\mathcal{L}_{1}}$ being an $\omega$-saturated expansion of an o-minimal ordered group, and if the structures $R_{\mathcal{L}_{0}}$ and $R_{\mathcal{L}_{1}}$ define (with parameters) the same sets of arity 2 , then they define the same sets in any arity.

Hence, if the maximality result for the collection of one-variable functions established in [3] and [10] could be transferred from the real setting to an $\omega$-saturated setting, the correctness of the conjecture of van den Dries and Miller would follow.

In their original form, the results of [3] actually hold not only for expansions of the reals but also for $\omega$-saturated structures. Let $R_{\text {an,exp }}$ be any model of the theory of $\mathbb{R}_{\text {an,exp }}$ (in the language $\mathcal{L}_{\text {an,exp }}$ with relational symbols for each subset of $\mathbb{R}^{n}$ definable in the real exponential field with restricted analytic functions), and let $R_{\text {an,Pow }}$ be its reduct to the language $\mathcal{L}_{\text {an,Pow }}$ (the sublanguage of $\mathcal{L}_{\text {an,exp }}$ with relational symbols for each subset of $\mathbb{R}^{n}$ definable in $\left.\mathbb{R}_{\text {an,Pow }}\right)$. Given a reduct $R_{\mathcal{F}}$ of $R_{\text {an,exp }}$, let $H\left(R_{\mathcal{F}}\right)$ denote the set of germs at $+\infty$ of one-variable functions definable in $R_{\mathcal{F}}$ with parameters (the set $H\left(R_{\mathcal{F}}\right)$ being viewed as a subset of (the Hardy field) $\left.H\left(R_{\text {an,exp }}\right)\right)$. In [3, Corollary 2] Kuhlmann and Kuhlmann state that if $R_{\mathcal{F}}$ is a proper reduct of $R_{\text {an,exp }}$ and if, at the same time, $R_{\mathcal{F}}$ is an expansion of $R_{\text {an,Pow }}$, then $H\left(R_{\mathcal{F}}\right)=H\left(R_{\text {an,Pow }}\right)$.

For two o-minimal structures over the reals, the local compactness of the real line ensures the equivalence between the fact of having the same germs of one-variable functions at infinity and the fact of defining the same subsets of $\mathbb{R}^{2}$. It is therefore natural to wonder if this property still holds for structures over a general real closed field.

The object of Section 2 is to show that this is not the case in general. We exhibit two o-minimal expansions of a common nonarchimedean real closed 
field that define the same germs at infinity of one-variable functions while not defining the same global one-variable functions.

The results in Section 3 are independent of those of Section 2 but are also motivated by the conjecture of van den Dries and Miller; furthermore, the techniques used in both sections are similar. We show that there are many different maximal polynomially bounded reducts of $\mathbb{R}_{\text {an,exp }}$ : the maximality of $\mathbb{R}_{\text {an,Pow }}$ remains open, but there is no hope for $\mathbb{R}_{\text {an,Pow }}$ to be the greatest element among the polynomially bounded reducts of $\mathbb{R}_{\text {an,exp }}$ (all this taken in the sense of definability).

\section{§2. Germs versus functions}

In this section, we present two o-minimal expansions of a nonarchimedean real closed field $\mathcal{R}$ that define (with parameters) the same germs of onevariable functions at infinity but that do not define the same global functions in one variable.

Definition 2.1. A function $f: \mathbb{R}^{n} \rightarrow \mathbb{R}$ is said to be a restricted analytic function if there is a function $F$ analytic in a neighborhood of $[0,1]^{n}$ such that $f(x)=F(x)$ for $x \in[0,1]^{n}$ and $f(x)=0$ for $x \notin[0,1]^{n}$.

Let $\mathcal{R}$ be the field of Puiseux series (i.e., the direct limit of all the fields of formal Laurent series in $T^{1 / d}$ as $d$ ranges over $\mathbb{N}$ ). Considering $T$ as an infinitesimal, $\mathcal{R}$ can be regarded as an ordered field extension of $\mathbb{R}$, the order on $\mathcal{R}$ being defined by

$$
\left(\zeta=\sum_{k=k_{0}}^{\infty} a_{k} T^{k / d} \wedge a_{k_{0}}>0\right) \Leftrightarrow \zeta>0 .
$$

Following [13, Section 2], one can extend any restricted analytic function $f: \mathbb{R} \rightarrow \mathbb{R}$ to a function $\tilde{f}: \mathcal{R} \rightarrow \mathcal{R}$. Let $U$ be an open neighborhood of $[0,1]$, let $F: U \rightarrow \mathbb{R}$ be an analytic function such that $\left.f\right|_{[0,1]}=\left.F\right|_{[0,1]}$, and consider $\zeta \in \mathcal{R}:$

- if $\zeta<0$ or $\zeta>1$, let $\widetilde{f}(\zeta):=0$;

- if $0 \leq \zeta \leq 1$, let $\widetilde{f}(\zeta)$ be the formal composite of $F_{a_{0}}$ and $\rho(\zeta)$ where

- $a_{0}$ is the constant coefficient of the development of $\zeta$,

- $F_{a_{0}}$ is the (converging) Taylor development of $F$ at $a_{0}$ (which exists since $\left.0 \leq a_{0} \leq 1\right)$,

$-\rho(\zeta)=\zeta-a_{0}$. 
(It is possible to extend in a similar manner a restricted analytic function of several variables; however, we need only the one-variable case in what follows.)

Definition 2.2. Let $f: \mathbb{R} \rightarrow \mathbb{R}$ be a restricted analytic function, and let $\tilde{f}: \mathcal{R} \rightarrow \mathcal{R}$ be its extension to the fields of Puiseux series described above.

We will denote by $\mathbb{R}_{f}$ the structure

$$
\mathbb{R}_{f}:=(\mathbb{R} ;<,+, \cdot, f),
$$

and by $\mathcal{R}_{f}$ the structure

$$
\mathcal{R}_{f}:=(\mathcal{R} ;<,+, \cdot, \widetilde{f})
$$

REMARK 2.3. The structure $\mathbb{R}_{f}$ is o-minimal. As noted in [13, Corollary 2.11], it is also an elementary substructure of $\mathcal{R}_{f}$; that is, if $\phi\left(x_{1}, \ldots, x_{n}\right)$ is a first-order logic formula in the language $\mathcal{L}_{f}=\{<,+, \cdot, f\}$ and $\left(a_{1}, \ldots\right.$, $\left.a_{n}\right) \in \mathbb{R}^{n}$, the property $\phi\left(a_{1}, \ldots, a_{n}\right)$ holds true when interpreted in $\mathbb{R}_{f}$ if and only if the property $\phi\left(a_{1}, \ldots, a_{n}\right)$ holds true when interpreted in $\mathcal{R}_{f}$.

Definition 2.4. Let $\kappa$ be the generalized power series

$$
\frac{1}{2}+\sum_{k=1}^{\infty} T^{k+(1 / k)}
$$

For $\zeta \in \mathcal{R}$, we will write

- $\zeta<\kappa$ if $\zeta<1 / 2+\sum_{k=1}^{K} T^{k+(1 / k)}$ for some $K \in \mathbb{N}$,

- $\zeta>\kappa$ if $\zeta>1 / 2+\sum_{k=1}^{K} T^{k+(1 / k)}$ for all $K \in \mathbb{N}$.

This defines a Dedekind cut on $\mathcal{R}$.

We chose $\kappa$ so that the 1 -type over $\mathcal{R}$ associated to this cut is not definable. In particular, if $\zeta \in \mathcal{R}$ and $\zeta<\kappa$ (resp., $\zeta>\kappa$ ), there is $\xi \in \mathcal{R}$ such that $\zeta<\xi<\kappa$ (resp., $\zeta>\xi>\kappa)$.

Definition 2.5. Let $\tilde{f}: \mathcal{R} \rightarrow \mathcal{R}$ be as in Definition 2.2. Under the notation of Definition $2.4, \mathcal{R}_{\left.f\right|_{\kappa}}$ will denote the structure

$$
\mathcal{R}_{\left.f\right|_{\kappa}}:=\left(\mathcal{R} ;<,+, \cdot\left(\left.\tilde{f}\right|_{[0, a]}\right)_{a<\kappa},\left(\left.\tilde{f}\right|_{[b, 1]}\right)_{b>\kappa}\right) .
$$

(For convenience, we identify any partial function $g: \mathcal{R} \rightarrow \mathcal{R}$ to a total function by setting $g(x)=0$ for $x$ outside of the original domain of $g$.)

We can now state the first result of this section. 
Proposition 2.6. For any function $g: \mathcal{R} \rightarrow \mathcal{R}$ definable in $\mathcal{R}_{f}$ (with parameters), there is a positive $\varepsilon \in \mathcal{R}$ such that $\left.g\right|_{(0, \varepsilon)}$ is definable in $\mathcal{R}_{\left.f\right|_{\kappa}}$.

Once this proposition is established, we will need to choose $f$ so that $\mathcal{R}_{f}$ defines strictly more sets than $\mathcal{R}_{\left.f\right|_{\kappa}}$ does.

Recall the following definition.

Definition 2.7 (see Le Gal [4]). A function $f: \mathbb{R} \rightarrow \mathbb{R}$ is said to be a strongly transcendental restricted $\mathcal{C}^{\infty}$-function if $f(x)=0$ for all $x \notin[0,1]$ and $f(x)=F(x)$ for all $x \in[0,1]$, where

- $F: U \rightarrow \mathbb{R}$ is a $\mathcal{C}^{\infty}$-function in some neighborhood $U$ of $[0,1]$, and

- given any tuple $x=\left(x_{1}, \ldots, x_{n}\right)$ of pairwise distinct elements of $U$, there exists a constant $C \in \mathbb{N}$ such that, for all $m \in \mathbb{N}$, the transcendence degree over $\mathbb{Q}$ of the $n(m+2)$-tuple

$$
\left(x_{1}, \ldots, x_{n}, F\left(x_{1}\right), \ldots, F\left(x_{n}\right), \ldots, F^{(m)}\left(x_{1}\right), \ldots, F^{(m)}\left(x_{n}\right)\right)
$$

is higher than $n(m+2)-C$.

Following [4], if $x$ denotes the $n$-tuple $\left(x_{1}, \ldots, x_{n}\right)$, the notation $j_{n}^{m} F(x)$ denotes the $n(m+1)$-tuple $\left(F\left(x_{1}\right), \ldots, F\left(x_{n}\right), \ldots, F^{(m)}\left(x_{1}\right), \ldots, F^{(m)}\left(x_{n}\right)\right)$; the notation $\operatorname{trdeg}\left(x_{1}, \ldots, x_{n}\right)$ denotes the transcendence degree of $x$ over $\mathbb{Q}$.

Proposition 2.8. Under the notation of Definitions 2.2 and 2.5, if $f$ is a restricted analytic function which is also a restricted strongly transcendental function, then the function $\widetilde{f}$ is not definable in $\mathcal{R}_{\left.f\right|_{\kappa}}$.

REMARK 2.9. Note that the assumption on $f$ made in the hypothesis of Proposition 2.8 is nonvacuous: [4, Proposition 2.2] ensures that there exist (many) restricted analytic, strongly transcendental functions.

Propositions 2.6 and 2.8 imply the following.

THEOREM 2.10. There exists a pair of o-minimal expansions of a common nonarchimedean field that do possess the same set of germs at infinity of onevariable definable (with parameters) functions but do not possess the same set of global definable (with parameters) one-variable functions.

Proof of Proposition 2.6. Let $g$ be definable in $\mathcal{R}_{f}$ with some parameters $\beta \in \mathcal{R}^{p}$.

Up to compositions with $\emptyset$-definable Nash bijection between $(0,1)$ and $\mathbb{R}$, we can find a $\emptyset$-definable function $G$ from $[0,1]^{p+1}$ to $\mathbb{R}$ such that $g(x)=$ $\widetilde{G}(\beta, x)$, where $\widetilde{G}$ is the interpretation of $G$ in $\mathcal{R}_{f}$ (see Remark 2.3). 
By the syntactic version of Gabrielov's theorem of the complement (see $\left[1\right.$, Corollary]), there is some $q \in \mathbb{N}$ and some set $X \subset[0,1]^{p} \times[0,1]^{2} \times[0,1]^{q}$ such that the graph of $G$ is $\pi(X)$, where $\pi$ denotes the projection on the first $p+2$ coordinate axes, and such that $X$ is described by a finite Boolean combination of formulas of the form

$$
P\left(y_{1}, \ldots, y_{p+2+q}, f\left(y_{1}\right), \ldots, f\left(y_{p+2+q}\right), \ldots, f^{(m)}\left(y_{1}\right), \ldots, f^{(m)}\left(y_{p+2+q}\right)\right)=0
$$

and

$$
Q\left(y_{1}, \ldots, y_{p+2+q}, f\left(y_{1}\right), \ldots, f\left(y_{p+2+q}\right), \ldots, f^{(m)}\left(y_{1}\right), \ldots, f^{(m)}\left(y_{p+2+q}\right)\right)>0
$$

for $P$ and $Q$ some polynomial with coefficients in $\mathbb{Z}$.

Let $\widetilde{X}$ be the interpretation of $X$ in $\mathcal{R}_{f}$, and let $\widetilde{X}_{\beta}$ be its fiber over $\beta$ (defined by $\left.\widetilde{X}_{\beta}=\left\{z \in \mathcal{R}^{2+q} ;(\beta, z) \in \widetilde{X}\right\}\right)$.

By definable choice (see [12, Proposition 6.1.2]), for $\varepsilon>0$ small enough, there is a definable function $\zeta:(0, \varepsilon) \rightarrow \widetilde{X}_{\beta}$ such that for all $0<x<\varepsilon$ one has $(x, g(x))=\pi^{\prime}(\zeta(x))$ (where $\pi^{\prime}$ denotes the projection $\left.\mathcal{R}^{p} \times \mathcal{R}^{2} \times \mathcal{R}^{q} \rightarrow \mathcal{R}^{2}\right)$. Up to taking an even smaller $\varepsilon$, we can assume that each component $\zeta_{i}$ of $\zeta$ is continuous. If for each $1 \leq i \leq 2+q$ we denote $\xi_{i}=\lim _{s \rightarrow 0} \zeta(s) \in[0,1]$, we can further shrink $\varepsilon$ so that each set $L_{i}=\zeta_{i}((0, \varepsilon))$ is either a singleton or an open interval and its topological closure lies entirely in one side or the other of the cut $\kappa$ (the side depending on whether $\xi_{i}>\kappa$ or $\xi_{i}<\kappa$ ).

Let $\Gamma$ be the graph of $g_{\mid(0, \varepsilon)}$. We now have that

$$
\Gamma=\pi^{\prime}(\zeta((0, \varepsilon))) \subset \pi^{\prime}\left(\widetilde{X}_{\beta} \cap \prod_{i=1}^{2+q} L_{i}\right) \subset \Gamma .
$$

Since, for each $i$, the topological closure of each $L_{i}$ lies in one side or the other of the cut $\kappa$, there is some $c_{i}$ such that

- either $\left(0 \leq c_{i}<\kappa\right.$ and $\left.\left(\forall x \in \mathcal{R},\left(x \in L_{i} \rightarrow 0 \leq x \leq c_{i}\right)\right)\right)$,

- or $\left(\kappa<c_{i} \leq 1\right.$ and $\left.\left(\forall x \in \mathcal{R},\left(x \in L_{i} \rightarrow c_{i} \leq x \leq 1\right)\right)\right)$.

Because the set $\widetilde{X}_{\beta} \cap \prod_{i=1}^{2+q} L_{i}$ is a Boolean combination of sets of vanishing and sets of positivity of polynomials in the functions $\left(z_{1}, \ldots, z_{2+q}\right) \mapsto z_{i}$ and $\left(z_{1}, \ldots, z_{2+q}\right) \mapsto f_{\mid L_{j}}^{(d)}\left(z_{j}\right)$ with coefficients in $\mathcal{R}$, it is definable in $\mathcal{R}_{\left.f\right|_{\kappa}}$. It follows that $g_{\mid(0, \varepsilon)}$ is definable in $\mathcal{R}_{\left.f\right|_{\kappa}}$.

Before proving Proposition 2.8, we need the following real version of it. 
Lemma 2.11. Let $f: \mathbb{R} \rightarrow \mathbb{R}$ be a restricted analytic function. Assume, furthermore, that $f$ is a strongly transcendental restricted $\mathcal{C}^{\infty}$-function. Consider $(a, b) \in \mathbb{R}^{2}$ with $0<a<b<1$. Then $f$ is not definable in the structure $\left(\mathbb{R} ; \leq,+,\left.\cdot f\right|_{[0, a]},\left.f\right|_{[b, 1]}\right)$.

Proof of Lemma 2.11. Suppose that $f$ is definable in $\left(\mathbb{R} ;<,+, \cdot,\left.f\right|_{[0, a]}\right.$, $\left.\left.f\right|_{[b, 1]}\right)$ with some parameters. Let $g(x)=f(a x)$, and let $h(x)=f(x+b(1-$ $x)$ ). By [1, Lemma 3], we can find some $p \in \mathbb{N}$, a finite collection of subsets $X_{\nu}$ of $[0,1]^{2} \times[0,1]^{p}$, and a finite collection $V$ of points in $[0,1]^{2} \times[0,1]^{p}$ such that

(1) the graph of $g$ is the union of the projections on the first two coordinates of $V$ and of $X_{\nu}$

(2) each $X_{\nu}$ is the intersection of the positivity set $P_{\nu}$ of a finite set $\Omega_{\nu}$ of functions, with the zero set $Z_{\nu}$ of a finite set $\Theta_{\nu}$ of functions, where each function in $\Omega_{\nu}$ and $\Theta_{\nu}$ is given as a polynomial with real coefficients in the functions $\left(z_{1}, \ldots, z_{2+q}\right) \mapsto z_{i},\left(z_{1}, \ldots, z_{2+q}\right) \mapsto g^{(d)}\left(z_{j}\right)$, and $\left(z_{1}, \ldots, z_{2+q}\right) \mapsto h^{(e)}\left(z_{k}\right)$

(3) for each $\nu$, the set $X_{\nu}$ is an analytic manifold of dimension 1 given near each of its points by the transverse intersection of analytic hypersurfaces defined by each function in $\Theta_{\nu}$; and

(4) the projection on the first two coordinates has full rank 1 when restricted to each $X_{\nu}$.

The projection of $V$ being finite, we can find some $c \in \mathbb{R}$ and $\varepsilon>0$ such that $(c-\varepsilon, c+\varepsilon) \subset(a, b)$ and such that the set $\left\{(x, y) \in \mathbb{R}^{2} ; c-\varepsilon<x<\right.$ $c+\varepsilon, y=f(x)\}$ is the image by the projection $\pi:[0,1]^{2} \times[0,1]^{p} \rightarrow[0,1]^{2}$ of an analytic manifold $\Gamma$ given on some open set $U \subset[0,1]^{2} \times[0,1]^{p}$ as the conjunction of $p+1$ transverse smooth hypersurfaces of the form

$$
\left\{z \in U ; P\left(z, j_{2+q}^{m} g(z), j_{2+q}^{m} h(z)\right)\right\}
$$

for some polynomial $P$ and so that $\pi_{\mid \Gamma}$ is a one-to-one submersion between $\Gamma$ and the graph of the restriction of $f$ to $(c-\varepsilon, c+\varepsilon)$.

Let $\gamma$ be the preimage of $(c, f(c)) \in \mathbb{R}^{2}$ by $\pi_{\mid \Gamma}$, and let $\beta$ be a tuple made of the coefficients involved in the different polynomials $P$ used to describe $\Gamma$ in $U$.

By the chain rule and an easy induction, we can find, for all $D \in \mathbb{N}$, a rational function $\Phi^{D}$ with rational coefficients such that

$$
j_{1}^{D} f(c)=\Phi^{D}\left(\beta, \gamma, j_{n+p}^{D+m} g(\gamma), j_{n+p}^{D+m} h(\gamma)\right) .
$$


Let $\eta$ be an $s$-tuple whose coordinates are all the different images of the coefficients of $\gamma$ by the map $x \mapsto a x$ and $x \mapsto x+b(1-x)$. Then for all $D \in \mathbb{N}$ there is a rational function $\Psi^{D}$ with rational coefficients such that

$$
j_{1}^{D} f(c)=\Psi^{D}\left(a, b, \beta, \gamma, \eta, j_{n+p}^{D+m} f(\eta)\right) .
$$

Since $c \in(a, b), c$ is not a coordinate of $\eta$. The function $f$ being strongly transcendental, there is $C \in \mathbb{N}$ such that for all $D \in \mathbb{N}$,

$$
\begin{aligned}
(s+1)(D+1)-C & \leq \operatorname{trdeg}\left(c, j_{1}^{D} f(c), \eta, j_{s}^{D} f(\eta)\right) \\
& \leq \operatorname{trdeg}\left(c, j_{1}^{D} f(c), \eta, j_{s}^{D+m} f(\eta), a, b, \beta, \gamma\right) .
\end{aligned}
$$

But by (2.1),

$$
\operatorname{trdeg}\left(c, j_{1}^{D} f(c), \eta, j_{s}^{D+m} f(\eta), a, b, \beta, \gamma\right)=\operatorname{trdeg}\left(c, \eta, j_{s}^{D+m} f(\eta), a, b, \beta, \gamma\right),
$$

so that

$$
(s+1)(D+1)-C \leq s(D+m+1)+\operatorname{trdeg}(c, \eta, a, b, \beta, \gamma)
$$

However, the latter inequality cannot hold for large integers $D$ : this is a contradiction.

Proof of Proposition 2.8. Generalizing Lemma 2.11 to $\mathcal{R}$ is an easy syntactic manipulation.

Suppose by contradiction that $f$ is definable in $\mathcal{R}_{\left.f\right|_{\kappa}}$. By finiteness of firstorder logic formulas, $f$ is definable in the structure $\left(\mathcal{R} ; \leq,+, \cdot,\left.\widetilde{f}\right|_{[0, a]},\left.\widetilde{f}\right|_{[b, 1]}\right)$ for some $a$ and $b$ in $\mathcal{R}$ with $0<a<\kappa<b<1$.

Let $\mathcal{L}_{f, g, h}$ be the expansion of the real ordered field language obtained by adding three extra functional symbols of arity 1 (denoted, without ambiguity, $f, g$, and $h$ ), let $\mathcal{L}_{f}\left(\right.$ resp., $\left.\mathcal{L}_{g, h}\right)$ be its reduct obtained by removing the symbols $g$ and $h$ (resp., the symbol $f$ ), and let $\mathcal{R}_{f, g, h}$ be the $\mathcal{L}_{f, g, h}$-expansion of the real closed field $\mathcal{R}$ in which $f$ (resp., $g$ and $h$ ) is interpreted by $\tilde{f}$ (resp., $\left.\widetilde{f}\right|_{[0, a]}$ and $\left.\left.\widetilde{f}\right|_{[b, 1]}\right)$.

We then have

$$
\mathcal{R}_{f, g, h}=\exists \beta\left((y=f(x)) \leftrightarrow \phi_{g, h}(x, y, \beta)\right),
$$

where $\phi_{g, h}$ is an $\mathcal{L}_{g, h}$-formula. 
We can add new existential quantifiers so that each atomic formula appearing in the formula $\phi_{g, h}(x, y, \beta)$ either is in the pure language of rings or is of one of the forms $v=g(u)$ or $v=h(u)$ for some variables $u$ and $v$.

Let $a$ and $b$ be two distinguished variables, and let $\phi_{f}(x, y, a, b, \beta)$ be the $\mathcal{L}_{f}$-formula obtained by replacing in $\phi_{g, h}(x, y, \beta)$

- each atomic formula of the form $v=g(u)$ by a formula of the form $(0 \leq$ $u \leq a \wedge v=f(u)) \vee v=0$, and

- each atomic formula of the form $v=h(u)$ by a formula of the form $(b \leq$ $u \leq 1 \wedge v=f(u)) \vee v=0$.

Then

$$
\mathcal{R}_{f} \models \exists a \exists b \exists \beta(0<a<b<1) \wedge\left((y=f(x)) \leftrightarrow \phi_{f}(x, y, a, b, \beta)\right),
$$

and since $\mathbb{R}_{f}$ is an elementary substructure of $\mathcal{R}_{f}$ (as noted in Remark 2.3),

$$
\mathbb{R}_{f} \models \exists a \exists b \exists \beta(0<a<b<1) \wedge\left((y=f(x)) \leftrightarrow \phi_{f}(x, y, a, b, \beta)\right),
$$

which contradicts Lemma 2.11.

REMARK 2.12. Note that the question of whether Hardy fields of germs at infinity of one-variable functions determine the structure was asked with the hope of combining [11, Lemma 4.7] and [3, Corollary 2]. In the example presented in this section, even though we could have replaced $\mathcal{R}_{f}$ by an $\omega$-saturated $\mathcal{L}_{f}$-structure, $\kappa$ and $\mathcal{R}_{\left.f\right|_{\kappa}}$ were chosen precisely so that the structure $\mathcal{R}_{\left.f\right|_{\kappa}}$ is not $\omega$-saturated.

Consider $\mathfrak{R}_{f,\left.f\right|_{\kappa}}$, an $\omega$-saturated elementary expansion of the structure

$$
\left(\mathcal{R} ;<,+, \cdot, \tilde{f},\left(\left.\widetilde{f}\right|_{[0, a]}\right)_{a<\kappa},\left(\left.\widetilde{f}\right|_{[b, 1]}\right)_{b>\kappa}\right) .
$$

No analogue of Proposition 2.6 holds for the reducts $\mathfrak{R}_{f}$ and $\mathfrak{R}_{\left.f\right|_{\kappa}}$ of $\mathfrak{R}_{f,\left.f\right|_{\kappa}}$ : there is a realization $\chi \in \mathfrak{R}$ of the type $\kappa$, and the germ at $\chi$ of the realization of $f$ is not the germ of a function definable in the structure $\mathfrak{R}_{\left.f\right|_{\kappa}}$, precisely by the analogue of Proposition 2.8.

\section{$\S 3$. No greatest element}

In this section, we show that there are infinitely many polynomially bounded structures $\left(\mathbb{R}_{\mathcal{F}_{n}}\right)_{n \in \mathbb{N}}$ which are pairwise distinct maximal reducts of the restricted analytic field with exponentiation (all this in the sense of definability).

But first, let us state precisely what we mean by in the sense of definability. 
Definition 3.1. Given two structures $\mathcal{M}_{0}=(M ; \cdots)$ and $\mathcal{M}_{1}=(M ; \cdots)$ on the same universe $M$, we say that $\mathcal{M}_{0}$ is a (strict) reduct, in the sense of definability, of $\mathcal{M}_{1}$ (or that $\mathcal{M}_{1}$ is a (strict) expansion, in the sense of definability, of $\left.\mathcal{M}_{0}\right)$ if $\mathcal{M}_{0}$ defines, with parameters, (strictly) fewer sets than does $\mathcal{M}_{1}$.

Note that the fact that $\mathcal{M}_{0}$ is a reduct, in the sense of definability, of $\mathcal{M}_{1}$ does not imply that $\mathcal{M}_{0}$ is a reduct, in the classical sense, of $\mathcal{M}_{1}$; note also that $\mathcal{M}_{0}$ can be a strict reduct of $\mathcal{M}_{1}$ in the classical sense without being a strict reduct in the sense of definability.

Definition 3.2. Recall that an expansion of the real field is said to be polynomially bounded if whenever $f$ is a one-variable definable function, $f(x)$ grows at most as fast as a polynomial function as $x$ goes to $+\infty$. (That is, there is some $d \in \mathbb{N}$ such that $\exists M,\left(x>M \rightarrow|f(x)| \leq x^{d}\right)$.)

Polynomial boundedness is an important dividing line among o-minimal expansions of the reals. The growth dichotomy theorem of [6] states that polynomial boundedness is a necessary and sufficient condition for an ominimal expansion of the real field not to define the exponential function. (Note that [2] ensures that, given an o-minimal expansion of the real field, one can always expand it further by adding the exponential, while keeping o-minimality.)

Definition 3.3. We denote by $\mathbb{R}_{\text {an }}$ the expansion of the real field by all restricted analytic functions (see Definition 2.1), by $\mathbb{R}_{\text {an,exp }}$ the expansion of $\mathbb{R}_{\text {an }}$ by the exponential function, and by $\mathbb{R}_{\mathrm{an}, \text { Pow }}$ the expansion of $\mathbb{R}_{\mathrm{an}}$ by all the power functions (functions $f_{r}: \mathbb{R} \rightarrow \mathbb{R}$ defined by $f_{r}(x)=x^{r}$ if $x>0$, $f_{r}(x)=0$ if $\left.x \leq 0\right)$.

The structure $\mathbb{R}_{\mathrm{an}}$ is o-minimal and polynomially bounded following important results from Khovaskii, Łojasiewicz, and Gabrielov (see [12, Introduction]) and its expansion $\mathbb{R}_{\text {an,exp }}$ is still o-minimal (as first proved in [14]). The structure $\mathbb{R}_{\text {an,Pow }}$ is a strict reduct, in the sense of definability, of $\mathbb{R}_{\text {an,exp }}$ but a strict expansion, in the sense of definability, of $\mathbb{R}_{\text {an }}$ (by [5]).

As recalled in the introduction, van den Dries and Miller conjecture in [15] that $\mathbb{R}_{\text {an,Pow }}$ is maximal among the polynomially bounded reducts of $\mathbb{R}_{\text {an,exp }}$ (all this in the sense of definability).

Relying on results from [4], we prove the existence of an infinite collection of $\left(\mathbb{R}_{\mathcal{F}_{n}}\right)_{n \in \mathbb{N}}$ of maximal polynomially bounded expansions of the real field which are strict reducts of $\mathbb{R}_{\mathrm{an}, \exp }$ (all this in the sense of definability). 
The ideas involved in the proof of this theorem are largely inspired by the techniques developed by Le Gal [4, Corollary 4.2].

First, recall the following.

ThEOREM 3.4 ([4, Theorem 1.2]). For each $f: \mathbb{R} \rightarrow \mathbb{R}$ strongly transcendental restricted $\mathcal{C}^{\infty}$-function, the structure $\mathbb{R}_{f}:=(\mathbb{R} ; \leq,+, \cdot, f)$ is o-minimal and polynomially bounded.

See Definition 2.7; note that in this section, contrary to Section 2, the function $f$ is not required to be restricted analytic.

The next result, also from [4], states that the set of strongly transcendent $\mathcal{C}^{\infty}$-functions is hard to avoid. Let $\mathcal{A}$ be the set of restrictions to $[0,1]$ of functions which are analytic in a neighborhood of $[0,1]$, with radius of convergence at least 1 at each point of $[0,1]$. The norm $\|g\|=\sup _{k \in \mathbb{N}, x \in[0,1]}\left(\left|G^{(k)}(x)\right|\right) / k$ ! (where $G$ is any analytic continuation of $g$ to an open neighborhood of $[0,1]$ ) turns $\mathcal{A}$ into a Banach space. Let $\mathcal{S}$ denote the set of strongly transcendental restricted $\mathcal{C}^{\infty}$-functions.

Proposition 3.5 ([4, Proposition 2.2]). Consider $\eta$ any function admitting a $\mathcal{C}^{\infty}$-continuation to an open neighborhood of $[0,1]$. Then the set $\mathcal{A} \cap(\eta+\mathcal{S})$ is comeager in $\mathcal{A}$.

As a corollary, we get the following.

Corollary 3.6. Let $\varepsilon:[0,1] \rightarrow \mathbb{R}$ be the function defined by $\varepsilon(x)=e^{-1 / x}$ if $0<x \leq 1$ and $\varepsilon(0)=0$. There is a function $g \in \mathcal{A}$ such that, for all $n \in \mathbb{N}$, the function $f_{n}: x \mapsto g(x)+n \varepsilon(x)$ is a strongly transcendental restricted $\mathcal{C}^{\infty}$-function.

Proof. The proof is straightforward. For each $n \in \mathbb{N}, \mathcal{A} \cap(-n \varepsilon+\mathcal{S})$ is comeager in $\mathcal{A}$. But a countable intersection of comeager sets is also comeager. Therefore, $\mathcal{A} \cap \bigcap_{n \in \mathbb{N}}(-n \varepsilon+\mathcal{S})$ is comeager in $\mathcal{A}$. In particular, the Baire category theorem implies that $\mathcal{A} \cap \bigcap_{n \in \mathbb{N}}(-n \varepsilon+\mathcal{S})$ is nonempty.

Let $g$ be in $\mathcal{A} \cap \bigcap_{n \in \mathbb{N}}(-n \varepsilon+\mathcal{S})$; then, for each $n \in \mathbb{N}, f_{n}: x \mapsto g(x)+n \varepsilon(x)$ is strongly transcendental on $[0,1]$.

THEOREM 3.7. There is a family $\left(\mathcal{F}_{n}\right)_{n \in \mathbb{N}}$ of collections $\mathcal{F}_{n}$ of functions definable in $\mathbb{R}_{\text {an,exp }}$ such that,

- for each $n$, the structure $\mathbb{R}_{\mathcal{F}_{n}}:=\left(\mathbb{R} ; \leq,+, \cdot,(h)_{h \in \mathcal{F}_{n}}\right)$ is a maximal polynomially bounded reduct of $\mathbb{R}_{\mathrm{an}, \exp }$ (in the sense of definability), and

- for each $n_{1} \neq n_{2}$, the structures $\mathbb{R}_{\mathcal{F}_{n_{1}}}$ and $\mathbb{R}_{\mathcal{F}_{n_{2}}}$ do not define the same sets. 
Proof. For each fixed $n_{0}$, note that $f_{n_{0}}$ is definable in $\mathbb{R}_{\mathrm{an}, \mathrm{exp}}$. By Zorn's lemma, we can now complete the singleton $\left\{f_{n_{0}}\right\}$ to get a maximal set $\mathcal{F}_{n_{0}}$ of functions definable in $\mathbb{R}_{\text {an,exp }}$ such that the structure $\mathbb{R}_{\mathcal{F}_{n_{0}}}:=(\mathbb{R} ; \leq$ ,,$\left.+ \cdot(h)_{h \in \mathcal{F}_{n_{0}}}\right)$ is polynomially bounded.

By cell decomposition, the first conclusion of Theorem 3.7 is now satisfied.

For the second conclusion of Theorem 3.7, suppose that $\mathbb{R}_{\mathcal{F}_{n_{1}}}$ defines $f_{n_{2}}$ with $n_{1} \neq n_{2}$. Then $\mathbb{R}_{\mathcal{F}_{n_{1}}}$ defines $f_{n_{2}}-f_{n_{1}}=\left(n_{2}-n_{1}\right) \varepsilon$, contradicting the polynomial boundedness.

REMARK 3.8. Note that, given $n \in \mathbb{N} \backslash\{0\}$ and $f_{n}$ as in Corollary 3.6, the structure $\mathbb{R}_{\mathrm{an}, f_{n}}$ (obtained by expanding the restricted analytic field by the function $f_{n}$ ) defines the exponential: we have produced infinitely many polynomially bounded reducts of $\mathbb{R}_{\text {an,exp }}$ but none of them is an expansion of $\mathbb{R}_{\mathrm{an}}$ (all this in the sense of definability). If van den Dries and Miller's conjecture were to be proven true, it would follow that $\mathbb{R}_{\text {an,Pow }}$ is the unique maximal polynomially bounded reduct of $\mathbb{R}_{\text {an,exp }}$ that expands $\mathbb{R}_{\text {an }}$ (all this in the sense of definability): if $\mathbb{R}_{\mathcal{F}}$ is a maximal polynomially bounded reduct of $\mathbb{R}_{\text {an,exp }}$ that expands $\mathbb{R}_{\text {an }}$ (in the sense of definability), then, by [5, Result 3.2] and maximality, $\mathbb{R}_{\mathcal{F}}$ defines all power functions and is therefore an expansion, in the sense of definability, of $\mathbb{R}_{\text {an,Pow }}$.

Note also that the presentation of each $\mathbb{R}_{\mathcal{F}_{n}}$ is, in a double way, not constructive: first, the existence of a function $g$ as in Corollary 3.6 relies on the Baire category theorem and is therefore nonconstructive; second, once $g$ is chosen, the existence of each collection $\mathcal{F}_{n}$ is also given in a nonconstructive way, as a consequence of Zorn's lemma. This raises questions about elementary equivalence or isomorphism (in a certain sublanguage $\mathcal{L}$ of $\mathcal{L}_{\text {an,exp }}\left(\right.$ conjecturally $\mathcal{L}_{\text {an,Pow }}$ )) of all these maximal structures, each seen

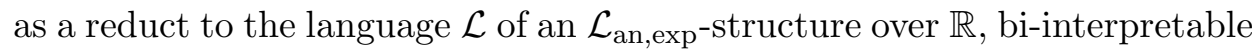
with the standard $\mathbb{R}_{\text {an,exp }}$ (in the spirit of [7, Theorem 2.1]).

Acknowledgments. The author's work was partially supported by the Institut de Recherche Mathématique de Rennes, by the Laboratoire de Mathématiques de l'Université de Savoie, and by the Agence Nationale de la Recherche "Singularités de Trajectoires de Champs de Vecteurs Analytiques et Algébriques" grant ANR 11-BS01-0009. 


\section{REFERENCES}

[1] A. Gabrielov, Complements of subanalytic sets and existential formulas for analytic functions, Invent. Math. 125 (1996), 1-12. MR 1389958. DOI $10.1007 / \mathrm{s} 002220050066$.

[2] M. Karpinski and A. Macintyre, A generalization of Wilkie's theorem of the complement, and an application to Pfaffian closure, Selecta Math. (N.S.) 5 (1999), 507-516. MR 1740680. DOI 10.1007/s000290050055.

[3] F.-V. Kuhlmann and S. Kuhlmann, Valuation theory of exponential Hardy fields, I, Math. Z. 243 (2003), 671-688. MR 1974578. DOI 10.1007/s00209-002-0460-4.

[4] O. Le Gal, A generic condition implying o-minimality for restricted $C^{\infty}$-functions, Ann. Fac. Sci. Toulouse Math. (6) 19 (2010), 479-492. MR 2790804.

[5] C. Miller, Expansions of the real field with power functions, Ann. Pure Appl. Logic 68 (1994), 79-94. MR 1278550. DOI 10.1016/0168-0072(94)90048-5.

[6] - Exponentiation is hard to avoid, Proc. Amer. Math. Soc. 122 (1994), 257259. MR 1195484. DOI 10.2307/2160869.

[7] A. Nesin and A. Pillay, Some model theory of compact Lie groups, Trans. Amer. Math. Soc. 326 (1991), no. 1, 453-463. MR 1002922. DOI 10.2307/2001873.

[8] B. Poizat, A Course in Model Theory: An Introduction to Contemporary Mathematical Logic, Springer, New York, 2000. MR 1757487. DOI 10.1007/978-1-4419-8622-1.

[9] S. Randriambololona, o-Minimal structures: Low arity versus generation, Illinois J. Math. 49 (2005), 547-558. MR 2164352.

[10] R. Soufflet, Asymptotic expansions of logarithmic-exponential functions, Bull. Braz. Math. Soc. (N.S.) 33 (2002), 125-146. MR 1934286. DOI 10.1007/s005740200005.

[11] L. van den Dries, Dense pairs of o-minimal structures, Fund. Math. 157 (1998), 61-78. MR 1623615.

[12] - Tame Topology and o-Minimal Structures, London Math. Soc. Lecture Note Ser. 248, Cambridge University Press, Cambridge, 1998. MR 1633348. DOI 10.1017/CBO9780511525919.

[13] L. van den Dries, A. Macintyre, and D. Marker, The elementary theory of restricted analytic fields with exponentiation, Ann. of Math. (2) 140 (1994), 183205. MR 1289495. DOI 10.2307/2118545.

[14] L. van den Dries and C. Miller, On the real exponential field with restricted analytic functions, Israel J. Math. 85 (1994), 19-56. MR 1264338. DOI 10.1007/BF02758635.

[15] - Geometric categories and o-minimal structures, Duke Math. J. 84 (1996), 497-540. MR 1404337. DOI 10.1215/S0012-7094-96-08416-1.

Galatasaray Ünivertisesi

Matematik Bölümü

Örtaköy/İstanbul

Turkey

serge.randriambololona@ens-lyon.org

serge.randriambololona@math.cnrs.fr 